\title{
(6) OPEN ACCESS \\ Growth of meibomian gland tissue after intraductal meibomian gland probing in patients with obstructive meibomian gland dysfunction
}

\author{
Steven L Maskin, Whitney R Testa
}

\begin{abstract}
- Additional material is published online only. To view please visit the journal online (http://dx.doi.org/10.1136/ bjophthalmol-2016-310097).
\end{abstract}

Dry Eye and Cornea Treatment Center, Tampa, Florida, USA

\section{Correspondence to} Dr Steven L Maskin, Dry Eye and Cornea Treatment Center, Tampa, 3001 Swann Avenue, Tampa, FL 33609, USA; drmaskin@tampabay.rr.com

Received 21 December 2016 Revised 29 March 2017 Accepted 2 April 2017 Published Online First 7 June 2017

\section{CrossMark}

To cite: Maskin SL, Testa WR. Br J Ophthalmol

2018:102:59-68

\section{ABSTRACT}

Purpose To investigate the impact of meibomian gland probing (MGP) on meibomian gland (MG) area from the upper lids of patients with obstructive meibomian gland dysfunction (o-MGD).

Methods Retrospective study comparing pre-MGP/ post-MGP non-contact infrared meibography results in patients with o-MGD, viewing signs of MG growth within total measurement field.

Results Post-MGP meibography of 34 lids (19 patients, $\geq 4.5$ to $\leq 12$ months' follow-up) showed $41.2 \%$ with MG growth. Ten lids had meibographies suitable for analysis, showing significant collective (116 glands) increase in mean individual glandular area (MIGA) of $4.87 \%$ $(p=0.0145)$. Four of 10 lids independently showed significant increase in MIGA, ranging from $10.70 \%$ to $21.13 \%(p<0.0001, p=0.0277, p=0.0292, p=0.0345)$, while six did not. At $>12$ and $<25$ months' follow-up, 16 lids (9 additional patients) had follow-up showing 25\% with signs of MG growth. Analysis of three lids showed a significant collective (33 glands) increase in MIGA of $11.19 \%(p=0.0004)$. Two of three lids independently showed significant increase in MIGA of $13.73 \%$ and $20.00 \%$ ( $p=0.0097, p=0.0001)$. Collectively, for all 13 analysed lids (149 glands), there was a significant increase of $6.38 \%$ in total glandular area $(p=0.0447)$ and a significant increase of $6.23 \%$ in MIGA $(p=0.0003)$. Conclusion MGP was associated with increased MG tissue area and growth of atrophied MGs as viewed on meibography. MGP provides unequivocal physical proof of a patent meibum outflow tract through the natural orifice, and may promote glandular growth in part by direct mechanical establishment of a patent duct/orifice system.

\section{INTRODUCTION}

Obstructive meibomian gland dysfunction (o-MGD) is considered the most frequent cause of evaporative dry eye in the world. ${ }^{12}$ o-MGD is associated with ageing ${ }^{34}$ and contact lens use, ${ }^{56}$ but is also associated with many causes of chronic ocular surface inflammation, including allergy and anterior blepharitis. ${ }^{278}$ Untreated, o-MGD will lead to atrophy of MGs characterised by infrared meibography (IR-M) changes, such as segments of discontinuous gland tissue, shortening of glands, and whole or partial gland dropout, leaving fading and poorly defined glands with an ultimate loss of all gland tissues. ${ }^{349}$ Symptoms develop from obstruction, causing elevated intraductal pressure, leading to lid tenderness (LT) and inflammation with subsequent lipid tear deficiency and dry eye. ${ }^{10-12}$
Treatment has classically focused on an anti-inflammatory approach using antibiotics and steroid along with lid hygiene using heat and lid margin cleansing with optional pressure to the lid to express glands and minimise lid margin inflammation and orifice obstruction. ${ }^{1314}$ Recently, new approaches to try and reverse glandular obstruction have included LipiFlow thermal pulsation ${ }^{15} 16$ and meibomian gland probing (MGP). ${ }^{10} 13$ 17-25 LipiFlow uses predetermined levels of external heat and pressure to try and force meibum through a duct and orifice obstructed by thickened meibum and intraluminal keratinised debris. ${ }^{16}$ In contrast, MGP inserts sterile stainless steel wire probes through the natural gland orifice to physically and directly unblock the orifice and ductal obstruction from thickened meibum and other non-fibrotic sources of obstruction. Importantly, MGP also relieves fixed obstructions, such as multifocal periductal fibroses, ${ }^{26} 27$ which are thought to occur along the length of the gland. ${ }^{19}$ The use of MGP allows equilibration of intraductal pressures on both sides of the obstruction with immediate and dramatic relief of LT and release of sequestered meibum with improved tear break-up time. ${ }^{1722-24}$ MGP has also been shown to restore meibum secreting gland functionality, significantly increasing the numbers of expressible glands per lid and improving quality and quantity of meibum lipid. ${ }^{19} 22$ A recent study, published ahead of print in 2017 from Massachusetts Eye and Ear Infirmary, showed over $91 \%$ of 70 probed lids resulted in subjective relief within 1 week, with no complications. $^{28}$

The goal of treating o-MGD should not be limited to symptom relief and stabilising age-associated or disease-associated gland atrophy, but rather to grow meibomian glands (MGs) and restore a full, functional, healthy and resilient MG lid population.

\section{MATERIALS AND METHODS \\ Study design and patient selection}

We conducted a review of the medical charts for 50 upper lids of 28 patients $(62.69 \pm 13.27$ years old with 2.5:1 female-to-male ratio) clinically diagnosed with o-MGD with at least one upper lid MGP, and a pre-MGP and post-MGP non-contact IR-M showing atrophy prior to MGP. Patient lids were grouped into less than or equal to 12 months or greater than 12 months' follow-up to reflect our clinical impression that the benefits of probing begin to diminish at approximately 1 year afterwards. All procedures adhered to the principles of the Declaration of Helsinki, and all patients 
provided informed consent. This chart study received an institutional review board (IRB) exempt review determination by an independent IRB.

o-MGD was clinically diagnosed by medical and ophthalmic history, as well as slit lamp examination, including evaluation of numbers of expressible glands and LT. Neither LT nor a cut-off number of expressible glands were required for a positive diagnosis of o-MGD. o-MGD was diagnosed clinically by slit lamp examination by signs including lid margin or tarsal hyperaemia, lid margin telangiectasia, thickening or irregularity, and MG orifice metaplasia, plus the presence or absence of LT in the setting of symptoms of o-MGD. LT was evaluated in a qualitative manner for this study as follows: one drop of $0.5 \%$ tetracaine hydrochloride (Bausch and Lomb, Tampa, Florida, USA) was placed in both eyes. The patient then looked into downgaze. Visualising through the slit lamp, a cotton tipped applicator (CTA) (Henry Schein, Melville, New York, USA) was then used to retract the upper lid to a position a couple of millimetres posterior to the limbus over the anterior sclera. Using the CTA, pressure was then applied to the skin surface of the upper lid posteriorly to the lash line over approximately the distal half of the MGs. This pressure was applied sequentially over the entire lid from right to left, and patients were asked if they felt tenderness or simply pressure. LT was recorded 'positive' for a lid if there was at least approximately one-third of the lid with tenderness. Focal LT over only a few consecutive glands did not assign LT for the entire lid.

The number of expressible glands was not required for the diagnosis of o-MGD. We know from probing that there are obstructions at different depths of the central duct; some ducts have obstruction just inside the orifice distal to the first acinus. Obstruction at this depth would lead to a gland without expressible meibum. Other ducts have obstruction proximal to at least the first acinus, such that there remains communication between acinus and orifice. This gland would still demonstrate expressible meibum even though the duct has proximal obstruction. If this proximal obstruction is not relieved, then the proximal gland behind the obstruction would show elevated intraductal pressure with subsequent atrophy leading to a short, truncated gland (see proposed classification diagram for o-MGD, figure 1). In other words, o-MGD may be present even if every gland is expressible. Fluorescein clearance test (FCT) and non-contact IR-M were also performed.

\section{Patient examinations and procedures} MG analysis

During routine slit lamp examination, and prior to MGP, the number of expressible MGs was counted by applying mild to moderate pressure with a CTA to the lid over the MGs to manually express meibum oil. The presence or absence of LT was also recorded. ${ }^{10}$

\section{Fluorescein clearance test}

Briefly, FCT was performed as previously described by placing one drop of $0.5 \%$ tetracaine hydrochloride (Bausch and Lomb) in both eyes, followed by placement of $5 \mu \mathrm{L}$ of $0.25 \%$ fluorescein sodium with $0.4 \%$ benoxinate hydrochloride (FluorBenox; Wilson Ophthalmic) into the inferior fornix of each eye by using an Eppendorf micropipette (Rainin Instrument, Oakland, California, USA). Wetting of $<3 \mathrm{~mm}$ on a 1 min duration test strip at $10 \mathrm{~min}$ was considered aqueous tear-deficient. ${ }^{29}$

\section{Non-contact IR-M}

Upper lid MGs were evaluated using non-contact IR-M with the Meibom Pen (Japan Focus, Tokyo, Japan) allowing for video capture of images. Dark or faded areas suggestive of acinar-ductular atrophy were noted and described as (1) whole or partial gland atrophy, (2) shortened and truncated glands, and (3) clumps or segments of discontinuous gland tissue. To perform upper lid meibography, the patient looked into downgaze, allowing for upper lid eversion for image capture. The strength and stability of the upper lid tarsal plate enabled repeat image capture on follow-up examinations of the same location with virtually the same camera angle while limiting distortion. ${ }^{3} 40$

\section{Intraductal MGP}

MGP was performed as previously described by the first author. The procedure was well tolerated by using the following anaesthetic protocol. Briefly, one drop of topical $0.5 \%$ tetracaine hydrochloride (Bausch and Lomb) was placed in the inferior fornix, followed by placing a bandage contact lens over the eye. Topical anaesthetic ointment consisting of $8 \%$ lidocaine with $25 \%$ jojoba in a petrolatum ointment base (Leiter's Compounding Pharmacy, San Jose, California, USA (no longer compounding); compounding currently being processed at O'Brien Pharmacy, Mission, Kansas, USA) was applied to the inferior lid margin. The eye was closed for $15 \mathrm{~min}$. One additional drop of topical tetracaine was then placed in the eye, and the patient was then positioned at the slit lamp. The MG orifices were then visualised and examined. A $1 \mathrm{~mm}$ long stainless steel sterile probe (Rhein Medical, St Petersburg, Florida, USA) was then inserted into each orifice, perpendicular to the lid margin using a dart throwing motion to find the angle of entry. Frequent 'pops and gritty sounds' were audible as periductal fibroses were released and resistance gave way allowing the probe to freely pass to and fro within the duct without resistance. ${ }^{10}$

\section{Data collection and analysis \\ Image analysis \\ Overview}

Everting upper lids for meibography could provide distorted views of MGs, in particular of the nasal and temporal lid. Therefore, the central half of the lid on meibographies was established by measurement with a millimetre ruler and used for analysis, typically defined by 9-16 glands. Our vertical dimension analysis guideline for total measurement field (TMF) was to use the posterior lid margin to the tarsal edge. Within this area, the TMF was outlined and used for analysis. The upper lids were excellent to evaluate for longitudinal follow-up study and preferred to the lower lid due to the dense tarsus providing a firm, sturdy structure to evert. This led to follow-up meibographies with only negligible change in shape or size of TMF, and consistent views of the same MGs. Using video meibography allowed us to use consistent camera angles and obtain numerous images to identify real changes in meibography findings over time. Without a dense tarsus, the eversion of the lower lid frequently caused diffuse distortion of MG images. For example, a mild pull or push of the lid horizontally or vertically may distend and lengthen, or compress and shorten, the lid, potentially affecting the size and shape of MGs and TMF, in particular with globe in upgaze, directly affecting the vertical height of the lower lid.

\section{Grading overall MG atrophy}

Post-MGP still-shot images from video meibography were then chosen to match with pre-MGP still images taken of the same view of the everted lid. All images were desaturated, then colour inverted in Photoshop. Analyses of the entire lid MG area were accomplished using ImageJ (http:// 


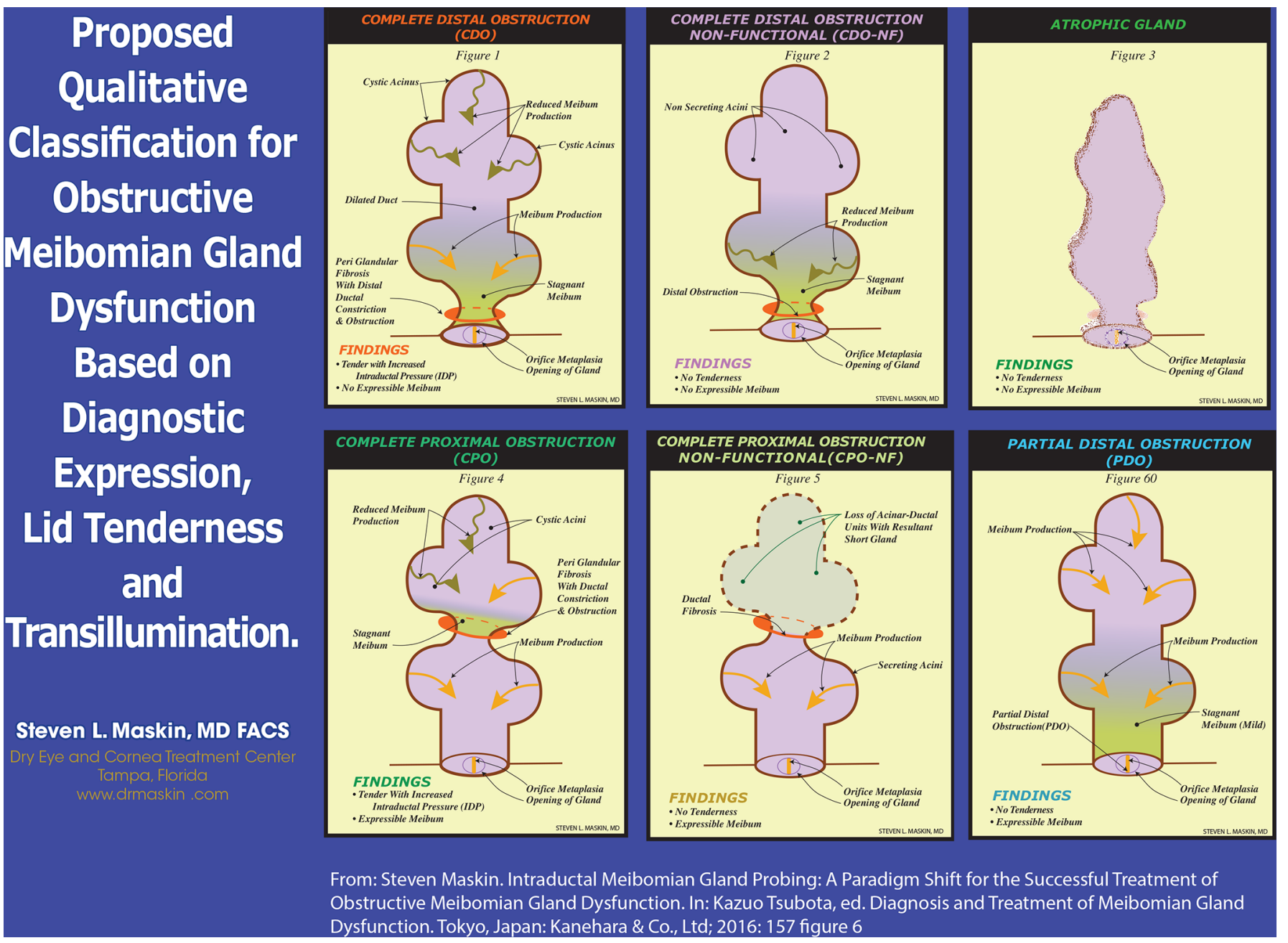

Figure 1 The top row of images represents a fixed obstruction (indicated by the orange ring around the gland duct) of the meibomian gland (MG) distal to all acini. This type of obstruction will initially present with lid tenderness (LT), as meibum is being produced without an escape, leading to the build-up of pressure behind the obstruction (this obstruction is termed complete distal obstruction, or CDO, as depicted in the first image of the top row). Eventually, the build-up of pressure will result in a loss of function of the gland (termed complete distal obstruction non-functional, or CDO-NF, as depicted in the middle image of the top row), ultimately resulting in MG dropout as the entire gland atrophies (top row, right). No meibum will be expressed from this gland. The first two images in the bottom row represent a fixed proximal obstruction (indicated by the orange ring around the more proximal gland duct) of the MG, in which one or more acini are in communication with the central duct and orifice. LT will be present, as meibum is being produced without an escape, leading to the build-up of pressure behind the obstruction. However, this classification of MGs will have expressible meibum, as the acini located distally to the obstruction still remains in communication with the central duct and orifice (this obstruction is termed complete proximal obstruction, or CPO, as depicted in the first image of the bottom row). Eventually, the build-up of pressure will result in a loss of function of the gland proximal to the obstruction, ultimately resulting in a truncated MG (middle image, bottom row). The final classification of partial distal obstruction (as depicted in the bottom row, right) is the result of a partial distally obstructed gland, such as orifice squamous metaplasia. This gland presents with no LT, as there is not a complete obstruction, and meibum is able to be expressed at the orifice. (From Steven Maskin, Intraductal Meibomian Gland Probing: A Paradigm Shift for the Successful Treatment of Obstructive Meibomian Gland Dysfunction. In: Kazuo Tsubota, ed. Diagnosis and Treatment of Meibomian Gland Dysfunction. Tokyo, Japan: Kanehara; 2016: 157 figure 6).

imagej.nih.gov/ij/; National Institutes of Health, Bethesda, Maryland, USA). In analysing overall grade of MG atrophy for the entire lid, each lid was classified as having $>0$ and $\leq 25 \%$ atrophy (grade 1), $>25$ and $\leq 50 \%$ atrophy (grade 2 ), $>50$ and $\leq 75 \%$ atrophy (grade 3 ), or $>75 \%$ atrophy (grade 4) by measuring the collective area of the glands compared with the entire area of the everted lid, using the polygon tool of Image J.

\section{Measuring per cent area of MG and total area of defined field}

Using the Microsoft Surface and Pen, with the image opened in ImageJ, the freehand and draw tools were used to outline the entire area of the TMF under evaluation as described above.
Each individual gland within the TMF was then measured for glandular area, and the ratio of individual and cumulative $M G$ area to the TMF was expressed as a percentage. The post-MGP MG area as a ratio to the TMF compared with pre-MGP was calculated and recorded as the percentage point difference. The growth in total MG area and individual glandular areas was then calculated by [(post-MGP area - pre-MGP area)/pre-MGP area] $\times 100$, and recorded (table 1$)$.

\section{Statistical analysis}

Statistical significance was set at $p<0.05$. The effect of MGP on glandular area was analysed via paired t-test with SD in 


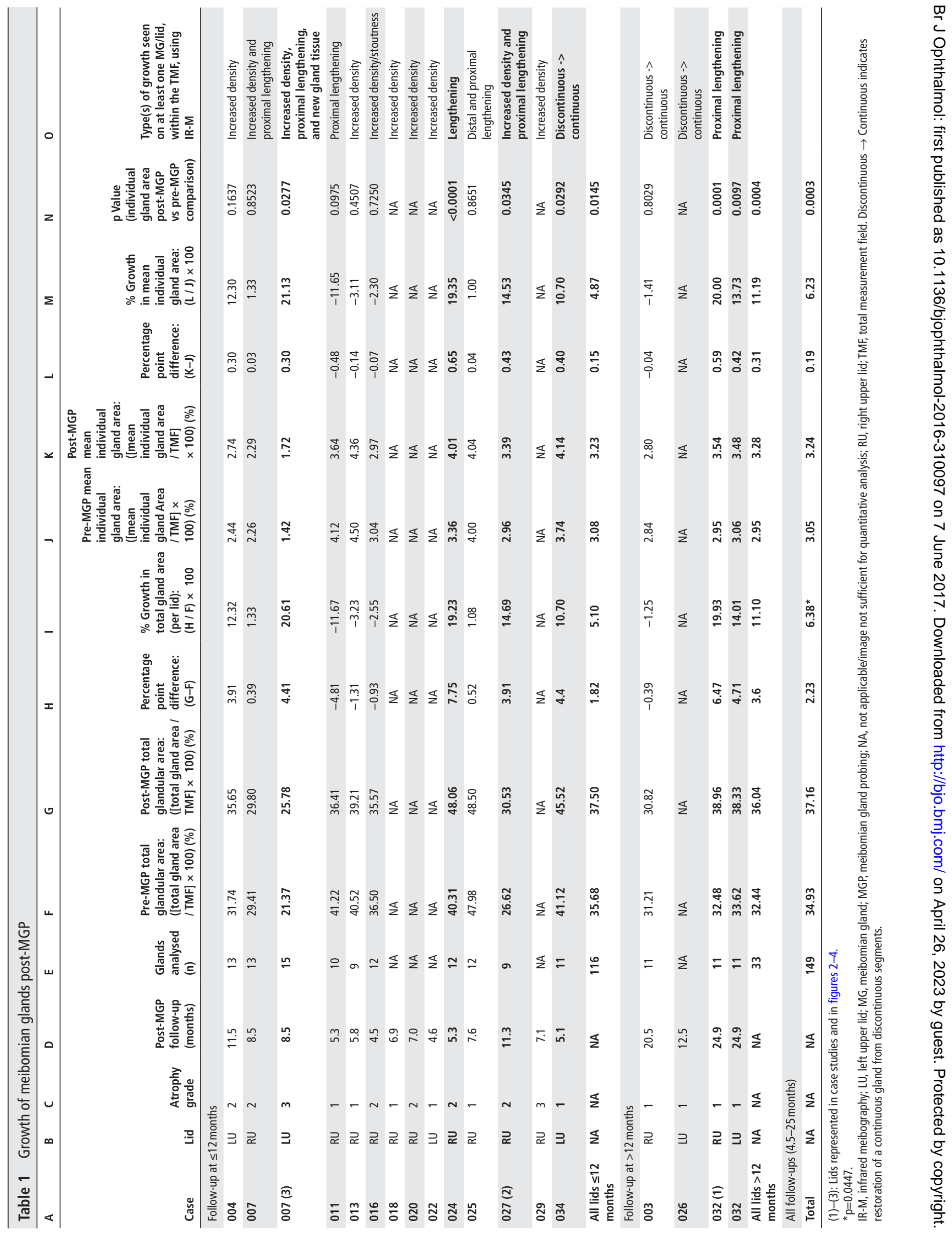


GraphPad Prism 7 for Windows (GraphPad Software, San Diego, California, USA).

\section{Representative cases}

Case 1

A middle-aged man was referred to this practice with a 5-year history of severe, excruciating pain, like firecrackers exploding in his eyes accompanied by symptoms described as gritty, sandy, scratchy and foreign body sensation with lid swelling, which began 5 years after LASIK. His symptoms were 'greater than 10

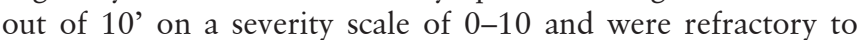
warm compresses with lid hygiene, preservative-free artificial tears, omega 3 fatty acids, punctal plugs, steroid drops, Restasis, Azasite, autologous serum, kineret, minocycline, LipiFlow and PROSE (prosthetic replacement of the ocular ecosystem). He was diagnosed with post-LASIK cornea neuropathy, put on Prozac and wore goggles while awake. Examination by the first author (SLM) led to additional therapies, including antibiotic ointment to lash roots, tea tree oil treatment for Demodex, topical antihistamine, punctal occlusion using thermocautery, oral cholinergic agents and ocular surface reconstruction with amniotic membrane for conjunctivochalasis. His lids showed o-MGD and were tender over the glands in each eye. He had expressible glands of 6 in the right upper lid (RU) and 3 in the left upper lid (LU). The right lower (RL) and left lower (LL) lids each showed 10. Preprobing meibography of the right upper lid showed an overall atrophy grade of 1 , with proximal atrophy and shortened, truncated glands. MGP of all orifices was performed on all four lids. Within 1 week after probing upper and lower lids, the lids were no longer tender and by 1 month expressible glands had increased to 10 (RU) and 17 (LU). The left lower lid increased to 16. Symptoms improved, and over the course of the next 2 years he has done progressively well overall with occasional irritation from episodes of anterior blepharitis, distichiasis, cellulose residue on lashes from artificial tears and stopping the use of topical antihistamine. He has been able to go to and enjoy the beach, and not wear his goggles for 4 hours per day. His Prozac dosage has been cut in half. His lids remained non-tender and expressible glands further increased to 17 (RU), 18 (LU), 21 (RL) and 19 (LL). Postprobing meibography showed statistically significant lengthening of glands of the upper lids, showing $20.00 \%$ growth for the right upper lid mean individual glandular area $(\mathrm{MIGA})(\mathrm{t}(10)=5.947, \mathrm{p}=0.0001)$ (figure 2, table 1 and online supplementary material video 1$)$.

\section{Case 2}

A middle-aged woman presented to this practice with a 1-year history of severe gritty, gravelly, sandy, foreign body sensation

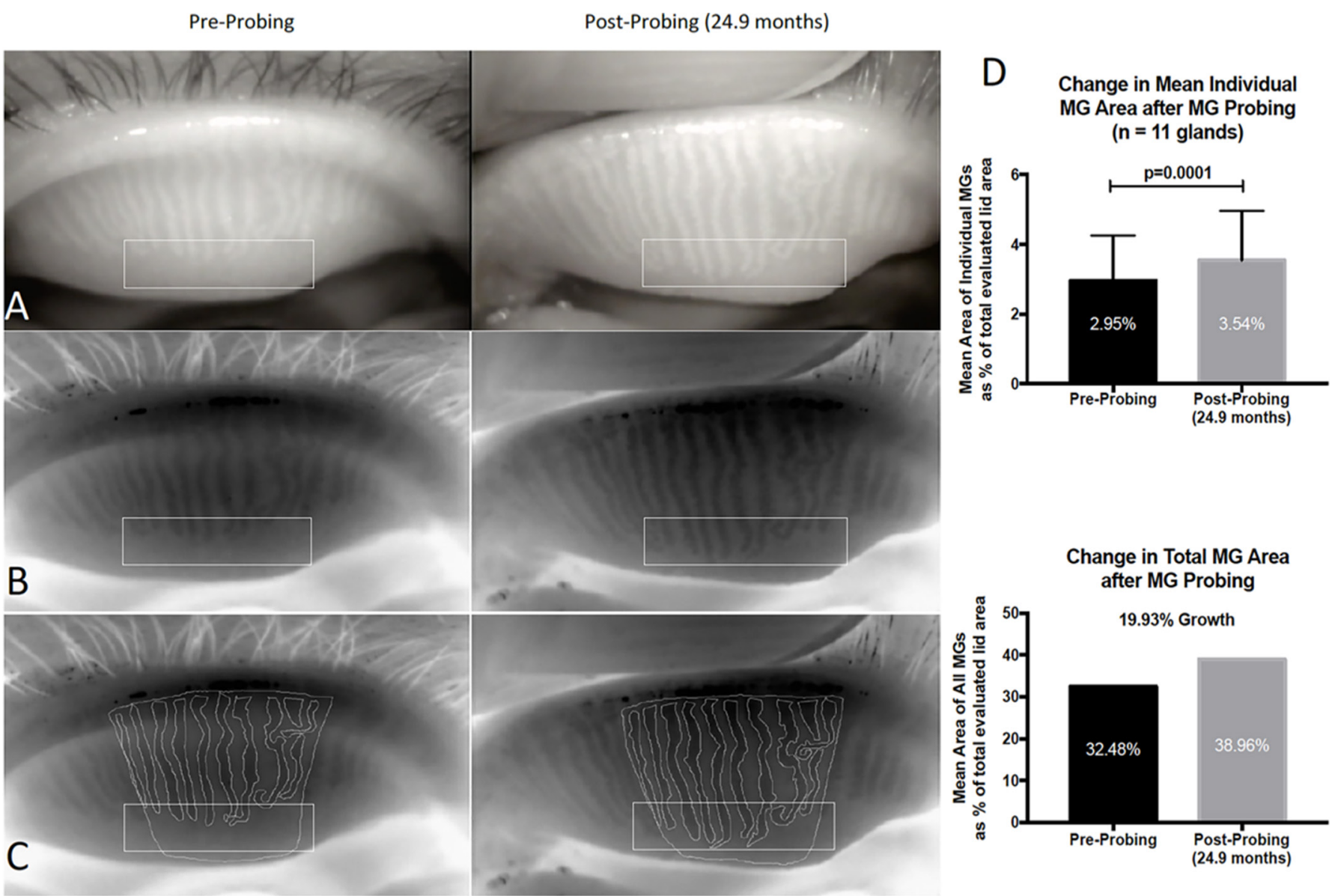

Figure 2 Case 1: The rectangles in A, B and C highlight the atrophic area in the total measurement field (TMF) where growth has occurred. (A) Preprobing and postprobing image capture from IR meibography video. (B) Preprobing and postprobing image captures after being processed through Adobe Photoshop simply to desaturate and invert the colour image. (C) Preprobing and postprobing traces of the TMF and each individual meibomian gland (MG). The preprobing images show proximal atrophy with shortened gland length. Postprobing shows an increased lengthening of these glands. (D) Statistical analysis revealed a significant $20.00 \%$ increase in mean individual glandular tissue postprobing $(t=5.947, p=0.0001)$, representing a $19.93 \%$ growth in total MG tissue area. 
with burning, itching, redness, sticky, photophobia, aching, heavy and tiredness with blurry vision. She had a permanent eyeliner procedure about the time her symptoms began. Her symptoms were 8 out of 10 on a $0-10$ scale. Warm compresses exacerbated her symptoms. Her symptoms were refractory to artificial tears and gels, steroid drops, omega 3 fatty acids, autologous serum and ice packs. Examination by the first author (SLM) led to additional therapies, including antibiotic ointment to lash roots, punctal occlusion by thermocautery and topical antihistamine. Azasite was tried but discontinued due to increased burning. Her lids showed o-MGD and were tender over the glands in all four lids. She had expressible glands of 2 (RU), 2 (LU), 1 (RL) and 5 (LL). Preprobing meibography of the right upper lid showed an overall atrophy grade of 2 , with whole gland atrophy with diffuse, fading glands, and proximal atrophy with shortening of glands. MGP of all orifices was performed on all four lids. Within 1 week of probing, symptom severity decreased to 2 out of 10 . Within 4 months, her lids were no longer tender and expressible glands increased to 6 (RU), 7 (LU), 8 (RL) and 8 (LL). Repeat meibography at 11.3 months postprobing showed statistically significant regrowth and increased density of faded, partially atrophic glands with proximal gland lengthening within the right upper lid, showing $14.53 \%$ growth in MIGA $(\mathrm{t}(8)=2.543$, $\mathrm{p}=0.0345$ ) (figure 3 and table 1 ).

\section{Case 3}

A middle-aged woman presented to this office with chronic red eyes for many years with superimposed acute exacerbation of redness with discharge every 2 months accompanied by heavy and tired eyes. Examination by the first author (SLM) led to tea tree therapy for Demodex, omega 3 fatty acids, punctal occlusion using thermocautery and ocular surface reconstruction using amniotic membrane. Azasite and doxycycline were also used. Her lids showed o-MGD and were tender over the glands in all four lids. There were zero expressible glands in each of her four lids. Preprobing meibography of the left upper lid showed an overall atrophy grade of 3 , with diffuse whole gland atrophy and marked fading of glands. MGP of all orifices was performed on all four lids. Within 1 month, she showed expressible glands of 4 (RU), 3 (LU), 4 (RL) and 2 (LL). Her right lower lid was no longer tender with the other three lids showing reduced tenderness. By 8.5 months, expressible glands increased to 14 (RU), 9 (LU), 9 (RL) and 8 (LL). Repeat meibography showed statistically significant regrowth and increased density of faded
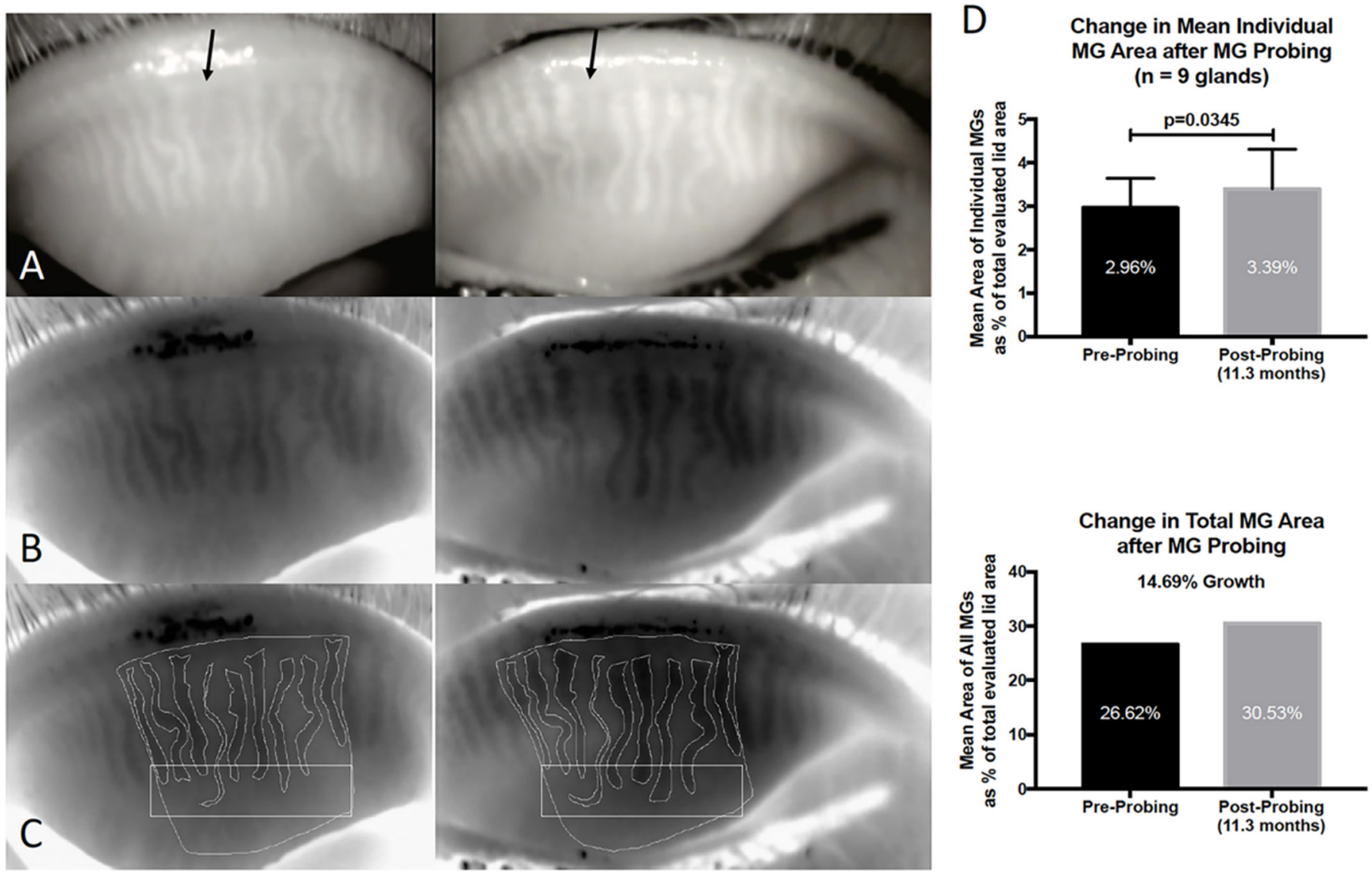

Figure 3 Case 2: (A) Preprobing and postprobing image captures from infrared meibography video. The arrows correspond to the same atrophied gland. (B) Preprobing and postprobing image captures after being processed through Adobe Photoshop simply to desaturate and invert the colour image. (C) Preprobing and postprobing traces of the total measurement field (TMF) and each individual meibomian gland (MG). The rectangles highlight an atrophic area in the TMF where growth has occurred. The preprobing images show diffuse, whole gland atrophy (both nearly absent and fading glands) and proximal atrophy with shortened gland length. Postprobing, we see an increased density and definition in the faded atrophic glands, as well as a lengthening of these glands. (D) Statistical analysis revealed a significant $14.53 \%$ increase in mean individual glandular tissue post probing ( $t=2.543, p=0.0345)$, representing a $14.69 \%$ growth in total MG tissue area. 

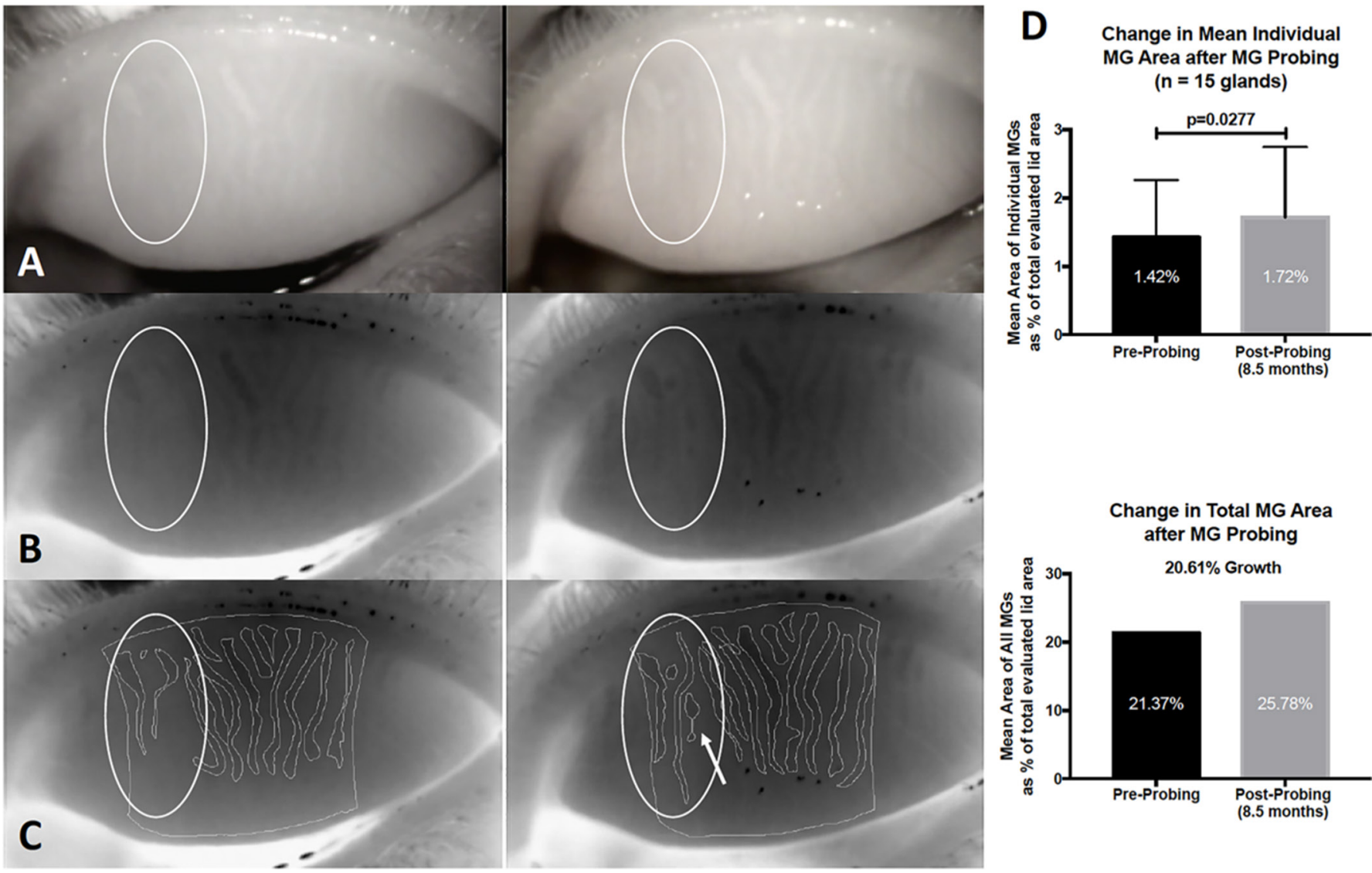

Figure 4 Case 3: The ovals in A, B and C highlight an atrophic area in the total measurement field (TMF) where growth has occurred. (A) Preprobing and postprobing image captures from infrared meibography video. (B) Preprobing and postprobing image captures after being processed through Adobe Photoshop simply to desaturate and invert the colour image. (C) Preprobing and postprobing traces of the TMF and each individual meibomian gland (MG). The preprobing images show diffuse, whole gland atrophy (both absent and fading glands) and proximal atrophy with shortened gland length. Postprobing, we see an increased density in the faded, atrophic glands, as well as new gland appearance (arrow inside oval), and lengthening of these glands. (D) Statistical analysis revealed a significant $21.13 \%$ increase in mean individual glandular tissue postprobing ( $t=2.456, p=0.0277$ ), representing a $20.61 \%$ growth in total MG tissue area.

atrophic glands with gland lengthening and a new gland noted within the left upper lid, showing $21.13 \%$ growth in MIGA $(\mathrm{t}(14)=2.456, \mathrm{p}=0.0277)$ (figure 4 and table 1 ).

\section{RESULTS}

\section{Growth in mean individual MG area after MGP}

Follow-up $>4.5$ months to $\leq 12$ months

The pre-MGP and post-MGP meibographies of 34 lids of 19 patients were examined, with 14 lids (41.2\%) presenting with signs of individual gland growth (mean follow-up time of $7.05 \pm 2.19$ months). Of these 14 lids, 3 had meibographies with light artefact or lid distortion, and 1 lid developed a hordeolum, limiting quantitative analysis (table 1). The remaining 10 lids of 9 patients had a collective total of 116 glands. Statistical analysis showed a significant increase in MIGA from $3.08 \% \pm 1.46 \%$ to $3.23 \% \pm 1.46 \%$, a $4.87 \%$ increase $(t(115)=2.483, p=0.0145)$. Furthermore, of the 10 lids evaluated, 4 individual lids (cases 007 LU, 024 RU, 027 RU and 034 LU) independently displayed statistical significance with an average increase of MIGA of $21.13 \% \quad(\mathrm{t}(14)=2.456, \quad \mathrm{p}=0.0277), \quad 19.35 \% \quad(\mathrm{t}(11)=6.475$, $\mathrm{p}<0.0001), \quad 14.53 \%(\mathrm{t}(8)=2.543, \mathrm{p}=0.0345)$ and $10.70 \%$ $(\mathrm{t}(10)=2.544, \mathrm{p}=0.0292)$, respectively. Six of 10 lids did not show significant, independent increase in MIGA.
Follow-up $>12$ months to $<25$ months

The pre-MGP and post-MGP meibographies of 16 lids of 9 patients were examined, with 4 lids $(25.0 \%)$ presenting with signs of individual gland growth (mean follow-up time of 20.71 \pm 5.07 months). Of these four lids, one had lid distortion, limiting quantitative analysis. The remaining three lids of two patients had a collective total of 33 glands. There was a significant increase in MIGA from $2.95 \% \pm 1.22 \%$ to $3.28 \% \pm 1.37 \%$, a $11.19 \%$ increase $(\mathrm{t}(32)=3.918, \mathrm{p}=0.0004)$. Furthermore, of the three lids evaluated, two individual lids (cases $032 \mathrm{RU}$ and $032 \mathrm{LU}$ ) independently displayed statistical significance with an average increase of MIGA of $20.00 \%(t(10)=5.947, p=0.0001)$ and $13.73 \%(t(10)=3.186$, $\mathrm{p}=0.0097)$, respectively.

Follow-up of all patients, collectively $<25$ months

The premeibographies and postmeibographies of a total of 50 lids of 28 patients were examined, with 18 total lids (36\%) presenting with signs of individual gland growth within 25 months post-MGP (mean follow-up time of $10.09 \pm 6.46$ months). Detailed analysis of 13 collective lids, consisting of 149 glands, revealed a statistically significant increase in MIGA from $3.05 \% \pm 1.41 \%$ to $3.24 \% \pm 1.44 \%$, a $6.23 \%$ increase $(\mathrm{t}(148)=3.687, \mathrm{p}=0.0003)$. 


\section{Growth in total MG area, per lid, after MGP}

For all 13 lids evaluated collectively, there was an increase in total glandular area, per lid, from $34.93 \% \pm 7.22 \%$ to $37.16 \% \pm 7.07 \%$, a $6.38 \%$ increase $(\mathrm{t}(12)=2.241, \mathrm{p}=0.0447)$. Total glandular area for the 10 lids collectively ( $\leq 12$ months' follow-up) as well as the 3 lids collectively ( $>12$ months' follow-up) did not reach statistical significance $(p=0.1521$ and $\mathrm{p}=0.5000$, respectively) (table 1 ).

\section{DISCUSSION}

Complete relief of all sources of gland obstruction may allow for and perhaps promote MG regeneration. Using conventional treatments for MGD such as heat and pressure, as with thermal pulsation, meibum release may be facilitated through the orifice from in front of or distal to possible fixed or periductal fibrotic obstruction (figure 1). This effect could yield lipid into the tear film with some improved clinical symptoms. However, using heat and pressure to force meibum out from behind an unyielding fixed obstruction, such as periductal fibrosis, may paradoxically increase intraductal pressure and inflammation behind the obstruction, with the possibility of increased symptoms and secondary proximal atrophy, analogous to squeezing a blemish. In contrast, MGP provides relief of all obstructions, fixed and non-fixed, and gives unequivocal positive proof of a patent duct and orifice. By relief of all obstructions, MGP appears to equilibrate intraductal pressures with immediate relief of LT, reduce inflammation and may create a microenvironment conducive to growth of MG tissue; such growth could be visualised in the clinic using IR-M.

It is well known in the literature that IR-M may demonstrate a loss of gland area, described as dropout, of MG structures. This dropout is generally accepted as representative of gland atrophy. Alternatively, IR-M may show an increase in gland area. If this increase in gland area is noted in previously normal-appearing glands, then in the clinical setting of o-MGD, it would be appropriate to suggest that this change in area is likely due to obstruction and increased intraductal pressure with dilated central duct and cystic deformation, leading to a widened appearing gland. In this setting, we have seen probing provides relief of obstruction leading to presumed equilibration of intraductal pressure and reduced gland area to more normal size. However, when the increase in gland area is in the setting of preprobing MG dropout and gland atrophy, we believe it is appropriate to use the term 'growth', as seen on IR-M.

We have presented three types of MG growth as indicated by meibography changes in this report: first, a lengthening of shortened glands reversing proximal atrophy; second, the partial restoration of faded glands with increased density and better definition; and third, the appearance of a new gland. We have also seen cases of restoration of a continuous gland from discontinuous segments of MG tissue. Successful MG growth is essential to fully restore full, functional, healthy and resilient MG lid populations. Healthy whole glands are preferable to functional yet partially atrophic glands. Whole glands can experience subsequent partial atrophy and still retain functionality, while already partially atrophic glands with obstruction may lead to whole gland atrophy. There is clinical value to the resilience of a restored gland.

The representative case examples in figures 2-4 showed increased MG tissue area and improvement from their baseline preprobing atrophy grades of 1,2 and 3. Case 1 showed only grade 1 preprobing atrophy, but had incapacitating, unrelenting severe pain perhaps related to history of LASIK-induced cornea neuropathy. Importantly, his MGs responded to probing with reduced LT, increasing his expressible gland count and showing significant gland lengthening leading to clinical relief. This demonstrates that the level of clinical symptoms may not always correlate with the grade of MG atrophy alone. The lack of improvement in symptoms using conventional therapy of heat and pressure (expression) in cases 1 and 2, and increased symptoms in case 2 , is notable. We have repeatedly seen this clinically and believe it is strongly suggestive of glandular fixed (periductal scarring) obstruction. In these cases, the heat and pressure bring more blood flow to an already inflamed gland with increased intraductal pressure. Probing breaks this cycle by relieving periductal scarring and patients can subsequently tolerate heat and pressure treatments.

The growth of atrophic glands is consistent with our unpublished clinical data for lids with atrophy grades 3 or 4 , as well as grades 1 or 2 , showing statistically significant improvement in signs and symptoms of o-MGD for both groups, including LT, lid functionality as a meibum secreting unit and the number of expressible glands. Collectively, for LT, a total of 541 tender lids were probed. By 1 week, 3-6 months and 1-year follow-up, $86.1 \%$ of $144,82.3 \%$ of 215 and $58.4 \%$ of 113 tender lids probed remained non-tender, respectively.

Additionally, there was a total of 271 non-functional lids (with four or less expressible glands per lid) that were probed. By 1 week, 3-6 months and 1-year follow-up, 93.8\% of 64, 92.2\% of 102 and $73.9 \%$ of 46 non-functional lids remained functional, respectively. In this cohort of probed, non-functional lids, the preprobing average of expressible glands was $2.4 \pm 1.5$ per lid (counting only up to 10 expressible glands per lid). By 1 week, 3-6 months and 1-year postprobing follow-up, there was a marked improvement in the number of expressible glands per lid to $8.7 \pm 2.1$ (264\% increase, $\mathrm{p}<0.0001), 8.5 \pm 2.2(253 \%$ increase, $\mathrm{p}<0.0001)$ and $7.0 \pm 3.0(193 \%$ increase, $\mathrm{p}<0.0001)$, respectively.

We also looked at the total number of expressible glands counted before and after probing, whereas prior testing stopped counting after 10 expressible glands were reached. In 25 preprobing non-functional lids, the average number of expressible glands was $2.8 \pm 1.1$ glands per lid, with a significant increase in the number of post probing expressible glands per lid to $14.4 \pm 6.4(412 \%$ increase, $\mathrm{p}<0.0001)$ at a mean follow-up of 2.4 months.

We believe earlier detection of symptomatic o-MGD with atrophy may allow for earlier intervention with relief of symptoms and reversal of atrophic MG changes. In asymptomatic patients with atrophic changes, MGP may promote MG growth, thus preventing onset of symptoms or possibly reversing subclinical symptoms only apparent after treatment. ${ }^{10}$

Our results show an overall incidence of $41.2 \%$ of lids showing individual MG growth between 4.5 and 12 months' follow-up. For all lids in the study, there was a collective $6.23 \%$ increase in MIGA, with individual lids showing up to $21.13 \%$ growth and a collective $6.38 \%$ increase in total glandular area per lid. Nearly $59 \%$ of lids did not show signs of growth, although these patients were clinically improved with relief of obstruction and resolved LT, as well as increased gland functionality (data not presented), consistent with previous reports. ${ }^{17}{ }^{22-24} 28$ If atrophic glands can grow, then why do we not see growth in all glands of all lids? One possibility may relate to the generally accepted finding that MGs undergo progressive atrophy with age and in the setting of comorbid diseases with surface inflammation and o-MGD. Probing has demonstrated the existence of intraductal fixed resistance in approximately $75 \%$ of upper lid glands, ${ }^{19}$ thought to be 
caused by periductal fibroses causing constriction and obstruction of the ductal lumen, able to be relieved post-MGP with improved symptoms and signs of o-MGD. However, the factors that lead to o-MGD continue to affect the glands postprobing, with eventual redevelopment of intraductal resistance and elevated intraductal pressure, which may act to suppress growth. These findings may explain the need to reprobe when clinically indicated, which in our experience is approximately on an annual basis. These factors may be systemic, such as in the setting of autoimmune disease, or preferentially found locally across the lid, such as nasally with allergy and aqueous tear deficiency, or temporally with conjunctivochalasis. Furthermore, there may be additional missing factors needed to promote growth. Together, with a restored patent central duct from probing, with adjunctive growth promoting factors in addition to eliminating comorbid diseases, we look to further improve on the $41 \%$ incidence of growth.

Another consideration is suggested by the results shown in column $\mathrm{O}$ in table 1 . These results show that each lid demonstrated signs of growth in at least one gland within the TMF. Signs of growth may be lengthening of a gland(s) or increased density of previously faded gland(s) showing dropout. Four of the six lids not showing statistically significant increased area, with $\leq 12$ months' follow-up, did show increased density of previously faded glands with dropout. This type of growth would therefore not be factored into an area-based calculation. Increased density may be thought of in three dimensions as representing increased acinar ductule units in two dimensionsviewed on IR-M at any point along the central duct.

We doubt experimental deviation greatly affected our results. It is possible that the manual tracing of the TMF and individual glands could introduce some experimental deviation. However, we believe our rigorous methodology in image evaluation, sample size and $p$ values (with significance set to $p<0.05$ ) supplemented with the SD have minimised these potential measurement effects. For example, when comparing the size of the TMF on preprobing and postprobing images, we found no statistically significant difference. We also have not noted day-to-day variability of IR-M gland area nor density for the same untreated individual.

One possibility to explain our findings is that the establishment of a patent orifice and duct permitted or perhaps promoted growth. This may be related to removal of the suppressive effect of elevated intraductal pressure on the proximal MG (proximal=deeper in lid). Alternatively, probing may activate MG stem cells with a direct mechanical intraductal stimulus. Although one paper concluded that the MG mouse stem cells lie at the circumference of each acinus, ${ }^{31}$ an abstract from 2003, using labelling studies, concluded that the circumference of each acinus had progenitor cells, and not stem cells, and the putative stem cells of the MG were more likely concentrated in the ductal epithelium. ${ }^{32}$ Other research suggests a mucoepidermal lineage is located within the central duct by the ductule orifice. ${ }^{33}$ It may be that stem cell activation is induced by probing the orifice/ duct tract, with debridement of cornified ductal epithelium. ${ }^{12}$ This could initiate proliferative effects with stem cell-induced transient amplifying daughter cells, leading to differentiation of ductal and acinar epithelium. ${ }^{12}$ Alternatively, the probing action may release stem cells to reimplant proximally, or deeper, in the gland. Close examination of distal gland portions, closer to the orifice, on meibographies suggests this portion may elongate postprobing, demonstrating the entire MG to be a dynamic epithelial gland able to grow under a favourable, non-suppressive environment. Future research is now warranted to study these post-MGP meibography changes in a randomised controlled clinical trial.
Correction notice This paper has been amended since it was published Online First. Owing to a scripting error, some of the publisher names in the references were replaced with 'BMJ Publishing Group'. This only affected the full text version, not the PDF. We have since corrected these errors and the correct publishers have been inserted into the references.

Contributors SLM and WRT planned and conducted the study, analysed the data, as well as drafted and revised manuscript. WRT performed statistical analysis. SLM is guarantor.

Funding This research received no specific grant from any funding agency in the public, commercial or not-for-profit sectors.

Competing interests SLM holds patents on the use of jojoba anaesthetic ointment, as well as instrumentation and methods (pending) for intraductal diagnosis and treatment of meibomian gland disease.

Ethics approval This retrospective chart review study was determined to be exempt from IRB review. It should be noted that this article does not contain any personal medical information about an identifiable living individual.

Provenance and peer review Not commissioned; externally peer reviewed.

Open Access This is an Open Access article distributed in accordance with the Creative Commons Attribution Non Commercial (CC BY-NC 4.0) license, which permits others to distribute, remix, adapt, build upon this work non-commercially, and license their derivative works on different terms, provided the original work is properly cited and the use is non-commercial. See: http://creativecommons.org/ licenses/by-nc/4.0/

(c) Article author(s) (or their employer(s) unless otherwise stated in the text of the article) 2018. All rights reserved. No commercial use is permitted unless otherwise expressly granted.

\section{REFERENCES}

1 Tomlinson A, Bron AJ, Korb DR, et al. The international workshop on meibomian gland dysfunction: report of the diagnosis subcommittee. Invest Ophthalmol Vis Sci 2011;52:2006-49.

2 Nichols KK, Foulks GN, Bron AJ, et al. The international workshop on meibomian gland dysfunction: executive summary. Invest Ophthalmol Vis Sci 2011;52:1922-9.

3 Arita R, Itoh K, Inoue K, et al. Noncontact infrared meibography to document agerelated changes of the meibomian glands in a normal population. Ophthalmology 2008;115:911-5

4 Arita $\mathrm{R}$, Itoh $\mathrm{K}$, Inoue $\mathrm{K}$, et al. Noncontact Meibography detects changes in meibomian glands in the Aging process in a normal Population and patients with meibomian gland dysfunction. Cornea 2009;28:S75-S79.

5 Arita R, Itoh K, Inoue K, et al. Contact lens wear is associated with decrease of meibomian glands. Ophthalmology 2009;116:379-84.

6 Arita R, Itoh K, Maeda S, et al. Association of contact lens-related allergic conjunctivitis with changes in the morphology of meibomian glands. Jpn J Ophthalmol 2012:56:14-19.

7 Guillon M, Maissa C, Wong S. Eyelid margin modification associated with eyelid hygiene in anterior blepharitis and meibomian gland dysfunction. Eye Contact Lens 2012;38:319-25

8 Fermon S, Zaga IH, Alvarez Melloni D. [Intraductal meibomian gland probing for the treatment of blepharitis]. Arch Soc Esp Oftalmol 2015;90:76-80.

9 Arita R, Itoh K, Maeda S, et al. Proposed diagnostic criteria for obstructive meibomian gland dysfunction. Ophthalmology 2009;116-2058-63.

10 Maskin SL. Intraductal meibomian gland probing relieves symptoms of obstructive meibomian gland dysfunction. Cornea 2010;29:8

11 Bron AJ, Tiffany JM. The contribution of meibomian disease to dry eye. Ocul Surf 2004:2:149-64.

12 Knop E, Knop N, Millar T, et al. The international workshop on meibomian gland dysfunction: report of the subcommittee on anatomy, physiology, and pathophysiology of the meibomian gland. Invest Ophthalmo/ Vis Sci 2011;52:1938-78.

13 Thode AR, Latkany RA. Current and Emerging Therapeutic Strategies for the Treatment of Meibomian Gland Dysfunction (MGD). Drugs 2015:75:1177-85

14 Geerling G, Tauber J, Baudouin C, et al. The international workshop on meibomian gland dysfunction: report of the subcommittee on management and treatment of meibomian gland dysfunction. Invest Ophthalmol Vis Sci 2011;52:2050-64.

15 Korb DR, Blackie CA. Restoration of meibomian gland functionality with novel thermodynamic treatment device-a case report. Cornea 2010;29:930-3.

16 Lane SS, DuBiner HB, Epstein RJ, et al. A new system, the LipiFlow, for the treatment of meibomian gland dysfunction. Cornea 2012;31:396-404.

17 Maskin SL. Intraductal meibomian gland probing relieves symptoms of obstructive MGD. Invest Ophthalmol Vis Sci 2009;50:1

18 Maskin SL, Kantor K. Intraductal meibomian gland probing with adjunctive intraductal microtube steroid injection (MGPs) for meibomian gland dysfunction (MGD). Invest Ophthalmol Vis Sci 2011;52:1. 


\section{Clinical science}

19 Maskin SL. Meibomian gland probing findings suggest fibrotic obstruction is A Major cause of obstructive meibomian gland dysfunction (O-MGD). Invest Ophthalmol Vis Sci 2012;53:1.

20 Wladis EJ. Intraductal meibomian gland probing in the management of ocular rosacea. Ophthal Plast Reconstr Surg 2012;28:416-8.

21 Dongju Q, Hui L. Jianjiang X: clinical research on intraductal meibomian gland probing in the treatment of patients with meibomian gland dysfunction. Chin J Optom Ophthalmol 2014;16:615-21.

22 Nakayama N, Kawashima M, Kaido M, et al. Analysis of meibum before and after intraductal meibomian gland probing in eyes with obstructive meibomian gland dysfunction. Cornea 2015;34:1206-8.

23 Ma X, Lu Y. Efficacy of Intraductal Meibomian Gland Probing on Tear Function in Patients With Obstructive Meibomian Gland Dysfunction. Cornea 2016;35:725-30.

24 Sik Sarman Z, Cucen B, Yuksel N, et al. Effectiveness of Intraductal Meibomian Gland Probing for Obstructive Meibomian Gland Dysfunction. Cornea 2016;35:721-4.

25 Qiao J, Yan X. Emerging treatment options for meibomian gland dysfunction. Clin Ophthalmol 2013;7:1797-803.

26 Cher I. Meibomian marginal dimples: clinical indicants of reactive pathogenic processes. Lass J, editor. Advances in corneal research: Selected Transactions of the World Congress on the Cornea. IV edn. New York: plenum Press, 1997:27-35.

27 Foulks GN, Bron AJ. Meibomian gland dysfunction: a clinical scheme for description, diagnosis, classification, and grading. Ocul Surf 2003;1:107-26.

28 Syed ZA, Sutula FC. Dynamic Intraductal Meibomian Probing: A Modified Approach to the Treatment of Obstructive Meibomian Gland Dysfunction. Ophthal Plast Reconstr Surg 2017.

29 Maskin SL. Effect of ocular surface reconstruction by using amniotic membrane transplant for symptomatic conjunctivochalasis on fluorescein clearance test results. Cornea 2008;27:644-9.

30 Arita R, Suehiro J, Haraguchi T, et al. Objective image analysis of the meibomian gland area. Br J Ophthalmol 2014;98:746-55.

31 Olami Y, Zajicek G, Cogan M, et al. Turnover and migration of meibomian gland cells in rats' eyelids. Ophthalmic Res 2001;33:170-5.

32 Lavker RM, Treet J, Sun T. Label-retaining cells (LRCs) Are preferentially located in the ductal Epithelium of the meibomian gland: implications on the mucocutaneous junctional (MCJ) Epithelium of the eyelid. Invest Ophthalmol Vis Sci 2003:44:1.

33 Call $\mathrm{M}$, Fischesser $\mathrm{K}$, Lunn $\mathrm{MO}$, et al. A unique lineage gives rise to the meibomian gland. Mol Vis 2016;22:168-76. 